\title{
Les mots du monde
}

\author{
Roland Eluerd
}

Université Paris III 5, Le Marquisat. 16190 Montmoreau, France

\begin{abstract}
Résumé. $\mathrm{Si}$ « le mot n'est pas la chose », pas de bosses sous le tapis de l'immanence. Mais, au-delà des mots, les emplois des mots sont têtus : le monde impose sa présence. Pour une lexicologie ouverte sur le monde, le premier acte est une prise en compte de l'usage ordinaire. Il laisse voir que le signe implique une certaine représentation de la chose, caractéristique de l'appréciation et de l'usage que nous en faisons. Cette représentation apprise et partagée force la clôture d'un espace seulement mental du signe. Ici, le schéma triadique du signe selon Peirce et le réalisme critique de Wittgenstein sont des guides utiles. Plusieurs exemples, tous concernant des noms très ordinaires, illustreront ce point de vue. Ils montreront comment le monde, à qui la porte de la langue est fermée, entre en quelque sorte par la fenêtre ouverte des sens figurés. Ainsi se dessinera la place d'une lexicologie des vocabulaires, moins soucieuse d'expliquer que de décrire.
\end{abstract}

\begin{abstract}
Words of the World. If "the word is not the thing", no bumps under the carpet of immanence. But, beyond words, the uses of words are stubborn: the world imposes its presence. For a lexicology open to the world, the first act is a consideration of ordinary usage. It shows that the sign implies a certain representation of the thing, characteristic of the appreciation and the use that we make of it. This shared and learned representation forces the closing of a merely mental space of the sign. Here, the triadic scheme of the sign according to Peirce and the critical realism of Wittgenstein are useful guides. Several examples, all concerning very ordinary names, illustrate this point of view. They will show how the world to which the door of the language is closed come in some sort by the open window of the figurative senses. Thus will be the place of a lexicology of vocabularies, less concerned with explaining than describing.
\end{abstract}

« Le lexique est au cœur de la réflexion sur la langue, ce qui peut s'expliquer par le fait qu'il organise l'essentiel des contenus exprimables linguistiquement et est, par là même, le point d'articulation entre pensée et langage. »

Cette phrase ouvre la présentation de la thématique consacrée au lexique dans l'appel à communication de ce $7^{\mathrm{e}}$ Congrès. Elle dit parfaitement le rôle des études lexicales dans une linguistique fondée sur l'immanence de la langue. Mais cette immanence m'a toujours paru être une limite gênante. Non pas tant gênante pour le lexique " point d'articulation entre pensée et langage », parce que ce lexique peut alors relever d'un univers mental dont le monde est exclu, mais gênante dès lors qu'on passe d'une lexicologie du lexique à une lexicologie des vocabulaires.

Aussi, je voudrais rendre au monde un peu de la place qui, me semble-t-il, lui est due. Il ne s'agit pas pour moi de prétendre proposer ce que Georges Kleiber appellerait une "xième théorie sémantique » (1999: 11). Il m'aurait fallu plus de moyens intellectuels et que je m'y sois employé depuis longtemps. Hic et nunc, c'est trop tard. Aussi, je n'offre ici qu'une invitation à une sorte de promenade lexicale, sans itinéraire de théorie ou de rhétorique, sans paragraphe et sous-paragraphe numérotés.

En premier lieu, je dois préciser que si l'immanence me chagrine, c'est parce que le débat de Socrate et Hermogène dans le Cratyle porte plus de questions que de réponses. C'est parce qu'il en va de même pour les thèses immanentistes : celles qui relèvent d'immanences internes au langage ou, comme plus souvent, celles qui relèvent d'immanences liées à une prétendue organisation du monde. Non pas évidemment le monde banal des usages ordinaires du langage, ce monde-là n'est pas considéré comme digne d'examen. L'organisation ne peut concerner qu'une dimension plus profonde de ce monde, une dimension cachée au commun des locuteurs, un méta-monde. Ce sera par exemple, la certitude des idées claires chez Descartes, celle d'une logique de distinction et de clarté chez Leibniz, la grammaire a priori de Husserl, l'innéisme de Chomsky, ou bien la doctrine figurale de l'exigence d'une organisation du monde pour que le langage soit lui-même organisé, telle qu'elle est exprimée dans le Tractatus logico-philosophicus de Wittgenstein. Autant d'exposés prestigieux de ces immanences qui relèvent toutes d'un réalisme non critique.

Tenir au strict domaine de la lexicologie et avancer que l'immanence y fait problème, ce n'est pas non plus prétendre à une quelconque originalité. Le renvoi saussurien de la lexicologie aux seuls rapports syntagmatiques et associatifs (Saussure, 1972 : 187) a été discuté dès le début des années 1950 par Georges Matoré (1953), et de nombreux travaux ont suivi où la lexicologie côtoyait le monde. Ainsi, celui des chemins de fer pour Peter Wexler

\footnotetext{
${ }^{1}$ roland.eluerd@free.fr
} 
(1955), de la vie politique et sociale pour Jean Dubois (1962), de l'aviation puis de l'astronautique pour Louis Guilbert (1965 et 1967), ou celui de la sidérurgie pour Roland Eluerd (1990).

Tous ces travaux, la doxa lexicologique immanentiste les range comme portant sur des mots de ce qu'elle désigne par le groupe nominal l'univers extralinguistique. Intéressant groupe nominal où l'adjectif n'est pas une simple épithète qualificative du nom mais, en quelque sorte, le capture ainsi que font les pseudo-épithètes de valeur attributive. De fait, au sens strict de l'expression, la linguistique définit l'univers comme... ce qui n'est pas la linguistique. L'univers évidemment manque de mots pour protester.

La même doxa accepte sans broncher qu'on définisse parfois le trajet sémasiologique selon un parcours allant « des mots vers les idées et les choses », et le trajet onomasiologique, « des idées et des choses vers les mots ». Je dis accepte parfois parce que les formules les plus courantes éliminent choses pour devenir à l'aller « des mots vers les idées ou les concepts » et au retour « des idées ou des concepts vers les mots ». À chaque fois : disparition pure et simple du monde et de ce qu'il pouvait avoir d'encombrant pour le trajet comme si l'objectif était d'alléger les bagages.

Or, tous les travaux que je viens de citer, et nombre d'autres, portent à la fois sur des mots et sur des choses du monde, des choses qu'il est mal commode de balayer sous le tapis de l'immanence parce que des noms comme locomotive, commune, avion ou haut-fourneau créent quand même d'assez grosses bosses de poussière. C'est pourtant sous ce tapis de l'immanence que les dictionnaires balayent ces mots parce qu'ils ne peuvent pas faire autrement. Ce ne sont que des dictionnaires. Ce qui est déjà beaucoup.

Quant à l'argument bien connu que dans un dictionnaire encyclopédique « les illustrations montrent les choses », donc le monde, il est quand même étrange. Les illustrations ne montrent pas les choses, elles montrent des images des choses. Certes les encyclopédies de toutes sortes sont appelées parfois « dictionnaires de choses », mais elles restent des dictionnaires de mots, des dictionnaires simplement ouverts à d'autres discours que ceux de l'usage ordinaire, discours scientifiques, techniques, de pratiques de toutes sortes, donc dictionnaires de mots quand même. Et dans ces dictionnaires, le monde demeure exclu.

Évidemment, si « Le signe linguistique unit non une chose et un nom mais un concept et une image acoustique » (Saussure, 1972 : 98), et si « le mot n'est pas la chose », pas de bosse sous le tapis. Mais l'évidence empirique de cette dernière sentence est telle qu'on oublie quand même qu'elle est plus analytique qu'empirique dans la mesure où mot et chose s'y définissent en miroir.

Je m'en remets ici à mon dictionnaire préféré, le Dictionnaire françois contenant les mots et les choses de Pierre Richelet. On conviendra que la recherche des noms du titre dans le dictionnaire lui-même est une démarche pertinente. Mot y est ainsi défini : «Tout ce qui se prononce, \& s'écrit à part. » Et ce n'est sans doute pas sans ironie que Richelet définit chose : "Mot qui se dit de tout ce qui subsiste, \& qui est au monde ». Cela avant de donner pour exemple une citation de Molière empruntée aux Précieuses ridicules : "Le mariage est une chose choquante. » Les longs relevés de Littré ou des dictionnaires contemporains ne retiennent pas mariage au premier rang des choses! Autre élément d'ironie, le parallélisme des deux définitions : mot, « tout ce qui se prononce, \& s'écrit à part », chose « tout ce qui subsiste, \& qui est au monde ». Sans oublier le début de la définition de chose : «Mot qui se dit de...»

En exploitant tout cela, on peut construire me semble-t-il une belle définition de chose : « tout ce qui est et peut être appelé chose ».

Objecter qu'on rencontre ici au mieux une tautologie, au pire une jonglerie verbale, ne rendrait pas compte de ce fait : chose n'est pas un pantonyme employé pour un autre mot - j'allais écrire pour «autre chose »-, chose est clairement employé pour « chose ». Ce que nous dit Richelet, c'est qu'il n'y a sans doute pas de mot plus «mot» que le mot chose. Alors que faire de la proposition « le mot n'est pas la chose »?

Pour reprendre les termes d'un article bien connu de Robert Martin, cette proposition n'en appelle à aucun univers de croyance, elle ne relève pas d'une analycité naturelle, ni d'une analycité conventionnelle, mais d'une analycité dont les termes sont déterminés par le système lui-même : "Sont analytiques les prédications que le système prévoit. L'analycité, en d'autres termes est déterminée alors par le système lui-même » (1985:54). La proposition « le mot n'est pas la chose » appartient au système de l'immanence et elle nous décrit ce système, mais elle ne nous dit rien des rapports entre le système et le monde.

Prenons une autre proposition emblématique de l'immanence : «le mot chien ne mord pas ». Je me garde ici d'aborder la formule « le concept de chien n'aboie pas », parce qu'elle est attribuée à tant d'auteurs qu'on s'y perd un peu, et parce que je veux, par prudence, n'étant pas philosophe, tenir sur le vocable mot. Si donc « le mot chien ne mord pas », pourquoi cela paraît-il bizarre de dire « le mot chien ne griffe pas » ? Et si cela parait bizarre de dire « le mot chien ne griffe pas », c'est sans doute que le mot chien mord quand même un peu. 
La prise en compte d'une relation notionnelle entre mordre et chien n'éclaire pas beaucoup la situation. Elle reste dans un espace linguistique qui est un espace mental. Une fois encore, elle conforte l'immanence par une définition de l'immanence. Cette pétition de principe ne permet pas de comprendre pourquoi mordre est attaché à chien, alors qu'un chat peut également mordre, ni pourquoi griffer est attaché à chat, alors qu'un chien a lui aussi des griffes. L'énergie que nous mettons dans l'expression «mordre dans la vie à pleines dents », l'énergie qu'Apollinaire injecte dans « Et nos baisers mordus sanglants», ou Baudelaire dans « Et le vers rongera ta peau comme un remords », comment pourraient-elles n'être que concept, idée, représentation mentale ?

Poser ces questions ne signifie évidemment pas refuser toute immanence. Une discipline soucieuse de sa spécificité commence très logiquement par balayer son terrain, puis elle le clôture et, dans cet enclos, elle conduit les recherches sans interférences qui la fondent. C'est une démarche épistémologique protocolaire. Mais pour le lexique, quand le protocole épistémologique fait que la clôture monte trop haut ou se fait trop épaisse, et que le contact avec l'extérieur se perd, cette perte devient considérable.

Déjà évoquée, la doctrine figurale du Tractatus de Wittgenstein donne l'une des images les plus achevées de cet enclos sans contact réel avec ce qui l'entoure. Mais Wittgenstein a lui-même énoncé la critique de ce qu'il a appellé ses « graves erreurs» $(1961: 112)$. Il l'a fait en passant de la conception figurale à une conception du langage comme praxis sociale. Cette conception a pour conséquence de forcer la clôture par la considération du rôle de l'usage, un usage appris et entretenu. Pour le lexique, c'est le sentiment somme toute très banal, que le mot tel que nous le donne par exemple un dictionnaire ou tel que nous pouvons l'avoir rencontré et appris, chaque locuteur le fait sien. Cela signifie que chaque locuteur interprète le mot et le réinterprète, sans qu'aucune barrière ne puisse interdire ou limiter ce processus. J'ai toujours trouvé très éclairante cette remarque qui se lit dans une lettre que Wittgenstein a adressée à Bertrand Russel : "Vous ne pouvez prescrire à un symbole tout ce qu'il peut servir à exprimer. Tout ce qu'un symbole peut exprimer (Wittgenstein emploie le défectif anglais can, "a la capacité de"), il peut l'exprimer (ici, il emploie le défectif anglais may, "est légitime que”) » (Monk, 1993 : 169).

La « légitimité » de l'expression ouverte du symbole ouvre sur la prise en compte de l'usage. Il est donc utile de mesurer comment la prise en compte du monde dans une lexicologie des vocabulaires participe de la prise en compte de l'usage ordinaire. Soit un exemple, la définition d'un nom très banal : pente.

Pente: «Obliquité par rapport au plan de l'horizon»(Trésor de la langue française informatisé, TLFi). "Inclinaison, déclivité d'un terrain, d'une surface par rapport à l'horizon » (Larousse en ligne). "Inclinaison d'un terrain ; toute déclivité en montée ou en descente » (Wiktionnaire). Ces définitions géométriques ne nous posent pas de problème de compréhension. Voilà bien un mot simple et clair. Lexicographie et géométrie marchent d'un même pas.

Dans les anciens dictionnaires, c'était plutôt avec la physique expérimentale ou la simple observation quotidienne que marchait la lexicographie. En effet, tous semblent avoir décidé de confier à l'eau la définition de pente. Quoi de plus visible en effet que l'écoulement de l'eau sur une pente ? Quoi de plus visible que, cette pente, l'eau ne la remonte jamais ? Bien entendu, les définitions ne font pas allusion explicitement à l'écoulement de l'eau, mais on lit très bien sa présence implicite dans l'absence d'une expression comme pente montante.

Richelet garde une certaine prudence : «Pante. Panchant. La manière d'être d'un corps qui va en panchant. » On voit certes rarement des corps aller penchant vers le haut, et du moins Richelet ne donne pas une image unique de pente descendante. C'est ce que font tous les autres dictionnaires. Pente : «Penchant qui va en descendant » écrit l'Académie, de 1694 ( $1^{\text {ère }}$ édition de son Dictionnaire) à 1798 ( $5^{\mathrm{e}}$ édition). Même approche chez Furetière, en 1690 : «Inclinaison ou descente d'un terrain d'un lieu haut vers un lieu plus bas. » Ainsi que chez Littré, deux

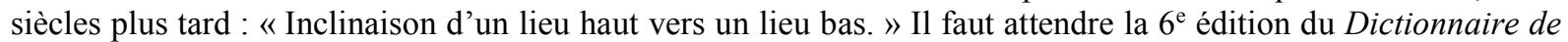
l'Académie, en 1835, pour que pente soit défini comme «l'inclinaison d'un plan », et la $8^{\mathrm{e}}$, en 1935 , pour que soit précisé « inclinaison d'un plan sur un autre plan ». Encore quelques années, et pente trouve la définition que lui réservent désormais les dictionnaires, celle «d'inclinaison par rapport à l'horizontale ». Soit un sens de pente où la pente peut descendre ou monter. On pourrait conclure qu'il n'y a là rien de bien intéressant sinon une définition plus précise où les dictionnaires de langue acceptent finalement les conseils logiques de la géométrie.

Cependant, cette logique se heurte au fait qu'il est légitime de considérer deux manières de vivre une pente, donc deux expériences linguistiques de pente : la pente que l'on voit, la pente où l'on marche. La pente que l'on voit est certes affaire de déclivité, une déclivité exprimée par nombre d'épithètes : pente douce, facile, forte, raide, abrupte. Cette pente appartient au monde naturel et, par les calculs ou les représentations graphiques, aux emplois de pente dans la réflexion humaine. L'autre pente, la pente où l'on marche, est, elle, affaire d'effort, de souffle et certaines des épithètes citées durcissent les mollets, meurtrissent les pieds. Elle appartient elle aussi au monde naturel. Si je plaçais pente dans le Dictionnaire des mots du sensoriel de Jean-François Bassereau et Régine Charvet-Pello (2011), l'évaluation sensorielle serait « visuel » pour le premier emploi et "kinesthésique ou corporel » pour le second. 
Rien de bien surprenant là encore, soit. Rien non plus qui puisse justifier que les dictionnaires distinguent deux noms homonymes pente. Encore moins qu'ils définissent l'un par « donne ou non le vertige » et l'autre par « coupe ou non le souffle ». Je reconnais que ces définitions semblent plus appartenir aux mots croisés qu'à la lexicographie. On voudra bien cependant considérer que ces ébauches de définitions ne seraient pas entièrement fausses et que, du moins, elles ne feraient pas l'impasse sur le monde. Et considérer également que s'il arrive que des exemples ou plus souvent des citations expriment ces valeurs mondaines, c'est justement parce qu'elles introduisent ce que je me résigne à nommer l'extralinguistique dans le corpus lexicographique, et que le monde $\mathrm{y}$ apparaît parfois avec plus de netteté et de force que dans des illustrations. Ainsi de cette citation du Père Goriot dans le $T L F i$ : « une pente si rude que les chevaux la montent ou la descendent rarement ».

Mais le plus intéressant se trouve dans les emplois figurés de pente. Je relève ceux que donne le même $T L F i$ : glisser sur la pente de la corruption, la pente de la décadence, la pente du libertinage; être sur une pente glissante, une pente savonneuse, une pente fatale; être sur une mauvaise pente, descendre la pente; sans oublier les emplois vieillis comme avoir une pente à l'avarice, une pente pour l'avarice ou le familier avoir la dalle en pente ! On voit clairement que tous ces emplois de pente participent de la définition des dictionnaires d'hier : la pente est une descente. Cela à un point tel que l'expression suivre sa pente, expression qui ne porte dans ses mots aucune précision quant à une obliquité positive ou négative de la pente qu'on suit, est toujours immédiatement interprétée en image de descente. C'est cette dominante interprétative de pente descendante qui donne son prix à la célèbre phrase de Gide dans Les Faux-Monnayeurs : «Il est bon de suivre sa pente, pourvu que ce soit en montant. $\rangle^{\mathrm{i}}$

On objectera l'absence de l'expression remonter la pente. Elle figure évidemment dans le TLFi comme dans tous les dictionnaires et j' aurais garde de ne pas en rendre compte. Certes, cette expression nous place en bas d'une pente, nous fait regarder vers le haut, donc vers une montée. Quand elle s'applique au sens propre, la pente est celle d'une colline, d'un versant qu'on vient peut-être de descendre. Elle porte alors avec elle les interprétations mondaines d'effort, de souffle que j'ai signalées. Mais l'emploi figuré est infiniment plus riche parce qu'il dessine deux mondes très différents dans les emplois de remonter la pente. Un premier monde qui est celui que j'appellerais le monde du «bien portant». Pour celui-ci, remonter la pente ne peut que dessiner une montée. On peut même le créditer de sympathie, de générosité quand il invite, plus ou moins fermement, celle ou celui qui est en bas de la pente à remonter la pente. Mais le « bien portant» qui avance cette invitation, s'il ose l'avancer, c'est souvent parce qu'il imagine mal que celle ou celui qui est en bas porte en son intime un autre monde, le monde de sa descente, un monde dont il conserve sans pouvoir les effacer des souvenirs, des images, des interprétations toutes liées à ces heures, à ces jours et ces nuits de la descente. Interprétants qu'il est facile de nommer : angoisse, obsession, peur, douleur, honte, désarroi... Interprétants dont on peut comprendre qu'ils rendent si difficile la remontée, l'idée même de la remontée. Interprétants qui font que remonter la pente dessine, désigne et signifie alors tout autre chose qu'une simple montée.

À cet égard, outre le fait que les dictionnaires d'hier avaient raison et que la meilleure définition de pente reste pour beaucoup d'emplois "sens de l'écoulement de l'eau », on mesure ici combien le monde fait irruption dans l'espace mental de la langue et en secoue l'immanence. Un monde qui est celui du vécu de chaque locuteur, un monde vécu et à vivre qui s'impose à la signification. Point d'articulation de la pensée et du langage, les mots sont également — parfois, souvent, surtout — la couture de la langue et du monde.

Conformément aux précautions prises quant à ma prudence devant toute théorisation, je me défausserai en disant simplement que la sémiologie de Charles Peirce me paraît offrir ici le meilleur éclairage. Pour Peirce, « un signe est une relation conjointe avec la chose dénotée et avec l'esprit » (1978:143). Autrement dit, la signification d'un nom ne peut pas être saisie dans la seule relation d'un signe, Peirce le nomme representamen, et de son objet, mais dans la médiation d'un troisième acteur de la sémiose dont le rôle est d'exprimer la relation signifiante. Ce troisième est lui même un signe que Peirce nomme interprétant. Conformément au schéma triadique, le representamen, l'objet et l'interprétant sont réels, mais seul le second, l'objet, existe (1978: 211).

On a souvent reproché à cette idée d'entraîner une suite infinie de renvois de signe à signe où se perdrait le sens et la raison. En réalité, la régression est interrompue par ce que Peirce appelle l'interprétant logique final. Cet interprétant n'est pas un signe mais un usage appris et entretenu dans le monde, un usage constitutif du monde : «Le pouvoir de décision revient à l'interprétant ultime, qui lui-même ne peut pas être un signe - puisqu'il est ultime et ne renvoie pas à un autre signe - et qui ne se situe pas à la fin des temps, mais dans le temps et en continuité avec lui : à l'interprétant logique final : l'habitude » (1978:220).

On croise alors les remarques où Wittgenstein, après avoir d'abord considéré que les significations étaient fixées selon des cadres logiques, les présentait comme reliées à des pratiques. « Nous demandons : Comment te sers-tu d'un mot, qu'en fais-tu ? - Cela nous apprendra comment tu le comprends » (1980:44). Et s'il fallait justifier le rapprochement connu mais, à mon sens insuffisamment exploité de Peirce et Wittgenstein ${ }^{\text {ii }}$ dans leur refus de fonder le signe sur une conception exclusivement psychologique, deux citations éclaireraient ce rapprochement. 
Wittgenstein : «Faites-vous enseigner la signification par l'usage » (1961 : 235). Peirce : « La signification d'un mot réside dans l'usage que l'on va en faire » (Deledalle, $1990: 41$ ).

À ces citations de Wittgenstein et de Peirce, j'ajouterais une autre citation qui sonne exactement dans le même ton : "C'est que nous sommes sans cesse dans l'occasion de prononcer qu'une chose est telle ; presque jamais dans la nécessité de déterminer ce que c'est qu'être tel. » Elle se lit dans l'article Encyclopédie de l'Encyclopédie, article rédigé par Diderot (Volume V: 635). Citer Diderot ici ne surprendra que ceux qui n'auraient jamais envisagé combien il a été un parfait témoin des vocabulaires de son temps et combien cette démarche, je dirais volontiers « terre à terre » de l'analyse des vocabulaires, a nourri ses réflexions sur le langage et la langue.

Pour les trois auteurs, le signe implique une certaine représentation de la chose, représentation caractéristique de l'appréciation et de l'usage que nous en faisons. Pour les trois « Comment parlons-nous? » est une interrogation inséparable de la question : « De quoi parlons-nous?»

Dans la remarque ci-dessus sur le monde du vécu de chaque locuteur, je disais : un monde vécu et à vivre qui s'impose à la signification. Les pronoms nous employés dans les deux questions précédentes invitent à ajouter maintenant : un monde partagé. Donc à voir ou, plus exactement, à rappeler, à survoler, comment des linguistes prennent en compte le contexte mondain.

Par exemple, comment, chez Oswald Ducrot, l'emploi d'un nom présuppose la réalité d'un monde d'objets (1972 : 236). Comment, chez John Searle, il n'existe aucun moyen d'éliminer la dépendance du sens littéral à l'égard du contexte (1982: 187). Comment, chez Antoine Culioli, les relations primitives de l'instanciation de la lexis constituent un système de représentation du contexte (Bastuji, 1982 : 43). Comment, chez Jacqueline Bastuji, qui prolonge en le discutant le modèle de Culioli, il y a dans les contraintes de la situation d'énonciation une détermination objective par la situation « extralinguistique » (1982 : 379). Comment, dans un article d'un prochain numéro de l'Information grammaticale consacré aux modèles de Culioli, une analyse sur ce que la modélisation sémantique peut apporter à la lexicographie, l'auteur conclut en écrivant que «l'interprétation possible ou non dépend entièrement de ce qui est construit dans le contexte du discours $\gg{ }^{\mathrm{iii}}$.

Comment enfin, dans l'article intitulé «La composante référentielle dans un manteau de laine et un manteau en laine », qu'Irène Tamba fit paraître en 1983, on peut lire qu'il eût été logique de penser que dans ces deux expressions la matière laine serait première par rapport à l'objet constitué manteau. Or l'instanciation de la lexis restitue un ordre inverse : «le caractère perceptif a manifestement prévalu. La matière est appréhendée consubstantiellement à l'objet qui l'incorpore et non de manière autonome » (1983:121). Bel exemple du genre de relation par quoi le langage « filtre » et non pas exclut le monde.

Accepter la prise en compte de semblables filtres contextuels est une manière d'accepter que le monde, le contexte mondain force la clôture de l'immanence. En voici quelques exemples, les uns liés à l'histoire du mot, les autres à la force, peut-être inattendue, des sens figurés.

Premier exemple, massif et très concret, le nom ouvrage. " Partie du haut-fourneau, haute de 0,5 à 1 mètre, audessus du plan des tuyères », donne le TLFi. Définition exacte, la base d'un haut-fourneau comporte trois parties : les étalages où la cuve de l'appareil se resserre pour concentrer le minerai de fer et le coke, l'ouvrage où cette masse est soumise à l'action des tuyères et le creuset où la fonte s'accumule avant la coulée. On pense alors très légitimement qu'il y a ouvrage parce que c'est l'endroit où la température est la plus intense et où s'accomplit l'œuvre, «l'ouvrage » de la réduction du fer en fonte. L'histoire du nom dit autre chose. Cette partie des hautsfourneaux de la fin du moyen-âge au XIX ${ }^{\mathrm{e}}$ siècle, fourneaux qui n'étaient pas « haut » comme ils le devinrent, mais qui étaient malgré tout des « hauts-fourneaux », relevait de tours de main, de méthode de construction dans l'assemblage des pierres et dans la préparation des joints dont les ouvriers fondeurs gardaient le secret et qui méritaient pleinement d'être nommé leur ouvrage. Ce que comporte l'ouvrage des hauts-fourneaux modernes, c'est donc l'histoire d'un métier et du savoir-faire des hommes qui l'accomplissaient. Un monde de travail.

Cela montre assez combien Pierre Lerat avait raison de souligner qu'une lexicographie terminologique doit «au préalable accepter une sémiotique non saussurienne de la langue » (1995: 168).

Je rapprocherai cette remarque sur ouvrage, des emplois figurés du nom bras. Ils sont nombreux à évoquer le travail : vivre de ses bras, de ses deux bras, en avoir plein les bras, ne pas avoir cent bras, soulever une charge à bras d'homme, à la force du bras, mettre de l'huile de bras, prendre à bras-le-corps ou à l'inverse rester les bras croisés. Pour tous ces emplois de bras, il s'agit bien d'entendre «travail ». Mais non pas sous le seul aspect de la force physique, comme on le réduit trop aisément. Il faut retenir également que ces emplois figurés de bras ont signifié « compétence », " savoir faire », « fierté », " dignité ». Derrière eux se dessine une histoire, voire une épopée des métiers où, au début de l'ère industrielle, comme l'a analysé François Jarrige, les machines furent nommées « tueuses de bras $»^{\text {iv }}$. 
Pour rester dans le domaine du corps humain, la lecture dans les dictionnaires des emplois figurés de dos, gorge, pas, etc. est singulièrement significative. On y découvre que l'expression qui résume le mieux les emplois du nom dos est sans doute : mal de dos. De fait, dorsalgie, lombalgie, lumbago, sciatique ont tout à voir avec en avoir plein le dos, avoir le dos large, l'avoir dans le dos, avoir sur le dos, être le dos au mur, etc. Le dos est certes fait pour porter le haut du corps. C'est ce que dit l'anatomie. Mais le vocabulaire figuré dit qu'il est surtout fait pour subir la charge des soucis, des infortunes, des trahisons. Des charges très mondaines, on en conviendra.

De même, la gorge humaine comme partie visible du cou semble n'exister que pour offrir sa fragilité à la violence. La poitrine peut avoir une cuirasse, la tête un casque, mais la gorge reste nue et les locutions la menacent : sauter à la gorge, couper la gorge, mettre un couteau sur la gorge, un coupe-gorge. Donc une valeur sémantique dominante pour presque tous les emplois de gorge figuré : " péril ». Une valeur sémantique largement mondaine, on en conviendra.

De même encore, le nom pas. «Pas. Le mouvement d'un animal mettant un pied devant l'autre pour marcher. Le pas d'un homme. le pas d'un cheval. » Cette définition est celle du premier Dictionnaire de l'Académie française. On la retrouve en ces termes ou des termes proches dans tous les dictionnaires, hier et aujourd'hui. Cela ne saurait surprendre. Mais elle ne rend pas compte d'une valeur sémantique mondaine, la valeur " mesure ». Cette valeur ne se trouve pas uniquement dans une expression vieillie comme vingt pas de long. On la trouve aussi dans $c$ 'est à deux pas, un pas de danse, doubler le pas, pas cadencé, faire le premier pas où sont mesurés temps, rythme, marche, argumentation. Elle est également présente dans les emplois de pas au sens de passage puisqu'il s'agit d'y mesurer un coût en temps et en risque : un mauvais pas, ou la nécessité de passer après : céder le pas. Ce que nous disent nombre d'emplois figurés de pas, c'est que l'être humain n'est pas qu'un bipède marcheur, c'est un arpenteur.

Le vocabulaire du corps humain n'est évidemment pas le seul à offrir de semblables pistes. Un seul exemple. Considérer la légitime colère féminine contre les comportements inadmissibles, voire criminels de certains " mâles », et leur dénonciation sous le vocable porc. Il est impossible de remplacer porc par cochon, plus encore par goret sans que la juste dénonciation y perde tout de sa force.

Le rôle que jouent ainsi les sens figurés pourra paraître surprenant. Comment concevoir que cette sorte de second degré du sens, a priori jugée encore plus éloignée du monde que le premier degré, celui du sens dit "propre », renverse en quelque sorte la problématique et, comme dans un combat à front renversé, facilite l'intrusion de l'usage et du monde dans l'immanence lexicographique? C'est pourtant ce que les sens figurés opèrent systématiquement. On ne doit pas en être surpris. Ici, je cite Henri Cottez, l'un des collaborateurs du Paul Robert : «Les métaphores sont autant de traceurs lexicaux permettant des observations d'ordre anthropologique, dans la mesure où elles opèrent une sélection de sèmes singulièrement révélatrice » (1982: 54). Par ce renversement, les sens figurés, à défaut d'entrer dans le sens linguistique par la grande porte, entrent en quelque sorte par la fenêtre.

Cela devrait inciter à ne pas lire trop vite les métaphores comme des images d'une réalité considérée comme virtuelle par une définition même de la métaphore. Mais n'est-elle pas importante cette remarque d'Alain dans un de ses Propos de littérature, justement sur la métaphore : « Le lac de Lamartine est un vrai lac ; observez comme les moindres traits font apparaître la loi des eaux et des rochers $\rangle^{\vee}$ ? De fait, la métaphore est tenue pour « virtuelle » moins par nature que par définition, c'est-à-dire qu'elle est tenue pour virtuelle quand elle est considérée à l'intérieur et de l'intérieur du système, donc par le système. Il semble d'ailleurs que cette virtualité de la métaphore paraisse plus facilement quand elle n'est qu'un trope. En revanche, la métaphore aristotélicienne est moins adaptable à cette définition par la virtualité. Irène Tamba et Paul Veyne l'ont clairement expliqué : « La metaphora aristotélicienne désigne, en premier lieu, la dénomination impropre d'un designatum » (1979 : 82). Ce designatum ne peut-on le rapprocher de l'objet de Peirce ? Quant à la " dénomination impropre », les deux auteurs précisent qu'elle est « obliquement translatée » (1979:83), elle l'est par un processus de l'intellect, qui peut être, lui, rapproché de l'interprétant.

Voyons deux exemples de lecture trop rapide de métaphores tenues pour virtuelles et excluant donc le monde : fibre, chez Diderot, et taureau de fer, chez Vigny.

Un ancien article de l'Information grammaticale (Eluerd, 1992), présente l'emploi de molécule et de fibre dans les passages où le Neveu parle de «la molécule paternelle » et avoue : "la fibre m'a manqué »vi. Les textes scientifiques traitant de la génération, à commencer par ceux de l'Encyclopédie, n'autorisent pas à conclure qu'on avait déjà affaire à des métaphores. Si cette fibre qui manque et cette molécule paternelle qui fait défaut gardent une part de fibreux ou de moléculaire pour proposer une explication de la paternité, on n'est pas devant un emploi figuré. Surtout si l'on entend les explications du docteur Bordeu à Mlle de l'Espinasse dans Le Rêve de d'Alembert : «Vous fûtes, en commençant, un point imperceptible, formé de molécules plus petites éparses dans le sang, la

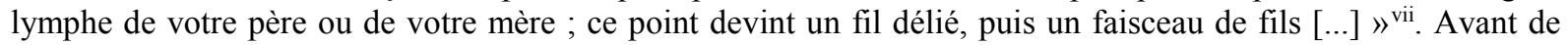
décider qu'il s'agit d'une métaphore, il est donc prudent de s'en tenir à l'intérêt que Diderot a pour ces vocabulaires spécialisés et pour la parlure du Neveu. Un intérêt qui participe de et à l'entrée des emplois de fibre et molécule 
dans l'usage ordinaire. Bien entendu, cette analyse ne pourrait être définitive qu'appuyée sur une histoire complète du vocabulaire de la physiologie naissante puis de son évolution. Et l'on peut mesurer que cette histoire ne serait pas totalement inutile au regard des progrès quasi quotidiens et des problèmes de la génétique actuelle.

Pour l'exemple emprunté à Vigny, la thèse déjà citée de Peter Wexler offre une aide solide au lecteur de La Maison $d u$ Berger. On connaît le passage où le poète regrette le développement du chemin de fer et veut en éloigner Éva : « Sur le taureau de fer qui fume, souffle et beugle, L'homme a monté trop tôt » (vers 78 et 79). Quelques vers plus loin, il évoque la « dragon mugissant qu'un savant a fait naître » (vers 90). Vigny a écrit ces vers le 11 juin $1842^{\text {viii }}$, donc un peu plus d'un mois après la catastrophe du train Versailles-Paris, dont il avait été témoin ainsi que Mme de Vigny. En 1842, le nom locomotive est connu. Pour autant, Peter Wexler montre que ce nom n'est qu'un vocable possible parmi machine à vapeur, machine locomotive, remorqueur, locomobile, etc. (1955:21). Et il indique également que les écrits publicitaires, les écrits protestataires, emploient un « langage lyrique et pré-mécanique » où l'on trouve « des éléphants, des taureaux, des coursiers d'airain... » (1955: 102, note). Quelles raisons aurait donc eues Vigny de préférer locomotive à remorqueur, taureau de fer à locomobile? Comment prévoir quel mot allait s'imposer? Comment entendrions-nous aujourd'hui locomobile, remorqueur ou pire dans les vers de La Maison du Berger ? La métaphore n'est donc qu'à peine une métaphore, bien plutôt une sorte de terme qui préserve l'avenir de la compréhension.

Les replis de cette compréhension, je les ai évoqués précédemment avec des noms du corps humain comme bras, gorge, pas. Je voudrais prolonger mes remarques en considérant la manière dont la part d'embarras des emplois de cœur dans Le Fils naturel de Diderot fait écho à l'embarras de l'article cœur dans l'Encyclopédie. Saisir leurs référents en termes de fragments du monde réel est évidemment possible. Mais ces fragments deviennent souvent des tissus anatomiques, d'où procède que Sénac, médecin consultant du roi et auteur de l'article cité, aura pu définir cœur : «l'âme matérielle de tous les corps vivants " ${ }^{\mathrm{ix}}$. Et cet embarras n'est pas l'affaire d'un moment: la définition de Sénac revoie à un ouvrage qu'il publia en 1749, l'article cœur se trouve dans le troisième tome de l'Encyclopédie, publié en 1753 et le Fils naturel est écrit en 1757. L'expression âme matérielle dit combien, quand, au milieu du XVIII ${ }^{\text {e }}$ siècle, la Nature est devenue une catégorie dominante, elle n'avait pas les arguments lexicologiques nécessaires pour que le nom nature prenne la place de sang ou de Dieu. C'est pourquoi la reconnaissance sociale qui clôt la pièce, tout autant que le mot qui termine son sous-titre : Les épreuves de la vertu, sont placés sous l'égide de l'expression le cœur droit. À la dernière scène, le fils reconnu, Dorval, reçoit cette admonestation de son père : «Je vous laisse une grande fortune. Jouissez-en comme je l'ai acquise. Ma richesse ne coûta jamais rien à ma probité $\|^{\mathrm{x}}$. Au fond, la société est passée de « Je rendrai mon sang pur comme je l'ai reçu » à « Je rendrai mon argent pur comme je l'ai reçu ». Le première sentence relevait du nom cœur, se suffisant à lui seul : « Rodrigue, as-tu du cœur ? ». Pour la seconde sentence, cœur doit s'adjoindre une épithète — je pense ici à la jeune Sidonie disant au capitaine Colette qui se piquait d'écrire : «Trop d'adjectifs ! —, le respect de la sentence du père et la nouvelle vertu seront l'ouvrage du cœur droit. Serions-nous en présence d'un ouvrage activé par un cœur de chair qui contiendrait quelque code génétique donnant la droiture ? Évidemment non. Mais alors il ne reste qu'un cœur figuré, lequel l'emporte sur le cœur anatomique. Avec, en corollaire, l'idée que le «vrai» cœur pourrait être ce cœur figuré.

Comme cette idée me tient «à cœur », c'est évidemment avec beaucoup d'intérêt que j'ai lu la récente thèse de Thomas Bertin sur la polysémie des noms de parties du corps humain (Bertin, 2018). Il y considère, entre autres noms, le nom cœur dont, écrit-il à juste titre, la polysémie est l'une des plus " éclatée » (2018: 17). Cette thèse expose une approche théorique de la polysémie où le mot «désigne un phénomène articulant un invariant sémantique (à un certain niveau d'abstraction) à une pluralité de sens (en contexte) » (2018:40). Avec une grande rigueur dans l'examen - et avec clarté dans le style, dernier point qui ne va pas toujours de soi pour ce genre d'exposé - Thomas Bertin conduit une analyse de tous les emplois de cœur, physiologique, psychologique, cognitif, méronymique, symbolique..., accompagnée de la prise en compte de multiples expressions courantes et de dominantes comme zone, courage ou bonté. Au terme de l'analyse, il propose « la formulation d'un invariant qui rend compte de l'identité sémantique du nom cœur par-delà la diversité de ses emplois (polysème) : En référence à une entité $E$, le nom cœur renvoie à une sous-entité $C$ à la fois intérieure à E et en relation avec l'extérieur de E. C'est au travers de cette relation que $C$ est le siège d'une valuation fortement positive sur un gradient qualitatif $\gg(2018: 320)$.

C'est, je dois l'avouer, un point que j'ai des difficultés à accepter ainsi. Non pas à cause de sa formulation très abstraite : il ne peut pas en être autrement et, dans l'ouvrage, un tableau explicite ces rapports. Mais parce que le monde, si présent dans toutes les analyses qui précédaient, me semble soudain s'effacer. Ou, plus exactement, semble effacé alors qu'il est toujours, de mon point de vue, très présent. Car enfin, en arrière-plan, voire en avantplan des entités $E$ et des sous entités $C$, il y a ce "gradiant qualitatif», cette pente inséparable du contexte mondain. Car enfin, souligner ainsi l'importance du registre axiologique dans l'invariant de cour, c'est dire à quel point l'axiologique devient l'épistémique, c'est dire à quel point les valeurs morales deviennent les valeurs de vérité. De ce fait, avancer, là encore, que le « vrai » cœur est le cœur figuré n'est pas totalement insensé. 
Peut-être ai-je tort? Non pas par refus de toute idée d'opérations linguistiques invariantes. Ce serait ridicule. Dans sa présentation d'une conférence donnée à la Société de linguistique de Paris ${ }^{\mathrm{xi}}$, Jean-Pierre Desclés, énumérait quelques-unes de ces opérations invariantes : «la prédication et la thématisation, la détermination et la quantification, la prise en charge énonciative de contenus... ». Ce sont là des opérations invariantes où le lexique est certes impliqué, mais sans que cette implication concerne tout ce que porte l'usage des mots. Et dans ses réserves sur l'aide que l'Intelligence artificielle peut apporter à la linguistique, Jean-Pierre Desclés montrait des limites qui me semble rejoindre mon propos. Ne disait-il pas que les processus de l'IA actuelle n'éclairent pas « les systèmes sémiotiques que sont les langues», "l'étude des différences sémantiques », "les variations paraphrastiques d'énoncés », la construction des dialogues « articulant différents référentiels »?

Cette articulation de différents référentiels, il me semble que la notion d'invariant, et toutes les notions proches comme celle de « signifié de puissance » ou celle de "prototype », notions ayant pour objectif d'assurer une part de stabilité au signe, contribuent à le solidifier dans l'immanence de représentations mentales qui procédent de modèles psychologiques pour lesquels le désordre ou la fluidité sont inacceptables. L'ouverture de la langue sur le discours comporte une belle occasion de libération par rapport à cette stabilité, mais comme toute une part de la pragmatique, elle reste souvent dans l'espace mental que cernaient nos deux mots de départ : pensée et langage. Bien entendu, les analyses inter-lexicales et intra-lexicales peuvent s'y développer à loisir et de manière très utile. Bien entendu, toute une lexicologie essentielle en procède.

À côté, je pense qu'il y a place pour quelque chose de moins organisé. Un mode de rassemblement qui ne soit pas un cadre solide, qui ne soit pas un système, peut-être même simplement une sorte de dynamique ouverte sur les flux de vie qui portent les sens. Une lexicologie qui prenne en compte l'usage, les jeux de langage, les définitions et les jugements. Une lexicologie — je ne parle pas de lexicographie —, une lexicologie qui évite de considérer comme inévitable ou nécessaire la délimitation d'une unité lexicale. Une lexicologie qui ne soit pas une lexicologie du lexique mais une lexicologie des vocabulaires telle que Robert-Léon Wagner souhaitait la voir se développer, il y maintenant 50 ans, quand il affirmait «la primauté des vocabulaires sur le lexique (1970: 18). Une lexicologie moins soucieuse d'explication que de description.

Je pense ici à ces mots qui sont restés si longtemps comme « en l'air ». On pourrait dire qu'il y avait le mot mais que la chose faisait défaut. Ainsi, pendant des siècles, on a fabriqué, trempé, affûté, vendu, acheté de l'acier. On a taillé, sculpté, là encore vendu, acheté du marbre ou du granit. Tout cela sans que ce fût de l'acier, du marbre ou du granit. Plus exactement, c'en était, par moment, parfois, par hasard sans qu'on sache ni pourquoi, ni comment. On a pensé en effet, pendant des siècles, que l'acier était un fer parfaitement pur. Les hommes des forges soumettaient le minerai fondu à l'action du feu, sans cesse et sans cesse, pour le purifier comme on purifiait l'or ou l'argent. Par hasard, comme je viens de le dire, il arrivait que le mélange du charbon et du fer tenait un pourcentage de charbon proche de celui qu'on appelle aujourd'hui le pourcentage de carbone dans l'alliage fer et carbone. À la fin du XVIII ${ }^{\mathrm{e}}$ siècle, l'acier a cessé d'être compris comme un fer parfaitement pur, pour devenir cet alliage, donc un fer impur. Le nom acier a trouvé sa « chose » mais cela ne l'avait pas empêché de vivre pendant des siècles sans elle et son prestige d'acier fer pur est demeuré intact passant sans remise en cause l'étape de l'impureté. On peut y lire une valeur sémantique majeure «perfection » qu'aucun dictionnaire ou invariant n'a pour tâche de relever.

De même, pendant des siècles, ont été travaillés des marbres qui, pour certains étaient de vrais marbres au regard de la définition géologique moderne. Pour autant, ils étaient nommés marbre tout comme beaucoup d'autres pierres qui n'étaient pas du vrai marbre mais qui se prêtaient bien au travail du tailleur, du sculpteur. C'est pourquoi les emplois actuels de marbre portent avec eux des sens et des interprétants mêlés, liés à la pierre, à la difficulté du travail, à la sculpture, à l'architecture, à l'Antiquité grecque et romaine, etc. On peut y lire une valeur sémantique majeure « beauté » qu'aucun dictionnaire ou invariant n'a pour tâche de relever.

Ou encore, la définition géologique de granit(e) qui procède des emplois de granit dans l'activité humaine depuis l'Antiquité. Ce nom désignait des roches graniteuses, devenues granit du fait de cette activité, mais aussi de vrais granit(e)s conformes à la future définition géologique. Granit, marbre et acier partagent le même destin : celui de mots qui existaient bien avant ce qu'ils ont fini par désigner. Celui de mots qui ont eu en quelque sorte la chance de « trouver» leur « chose ». Voilà des travaux de lexicologie des vocabulaires possibles : l'étude du vocabulaire du marbre, du vocabulaire du granit.

Et ces champs de vocabulaires à décrire sont nombreux. On peut commencer par considérer toutes les langues spécialisées. Que savons nous des vocabulaires de métallurgies comme celles du zinc, de l'aluminium, du plomb, des aciers spéciaux, des métaux rares ? Que savons-nous de l'usage des vocabulaires des fluides, des bâtis, du tourisme, de la dépendance et de l'âge, de la comptabilité, des finances, du « bio », des huîtres, des volailles, des jardins en général mais aussi des haies ou des feuilles ou des fruits, des aiguilles, des bornes, voire de la simple géométrie avec des mots comme pente, cercle, droite, point, ou du vocabulaire de la médecine au-delà des travaux de Bernard Quemada qui concernaient le période 1600-1710 ${ }^{\text {xii }}$ ? Bien sûr, nous disposons de nombreux glossaires numérisés, riches, précis et faciles d'accès. Bien sûr, tous ces glossaires sont parfaitement au point pour nous 
donner la définition de chaque terme, et qui connaît la discipline y trouve ce dont il a besoin. Mais quand on passe des termes aux mots, donc aux dictionnaires, des failles paraissent et cela dès les sens propres.

On peut voir, par exemple, comment les dictionnaires s'y retrouvent, ou plutôt cherchent à s'y retrouver, pour définir le nom cercle entre disque, la surface, et circonférence, la limite. À chaque fois, les sens figurés en laissent entendre plus. Que ce soit pour le cercle de famille qui « applaudit à grands cris », comme chacun sait depuis Hugo, ou ce cercle tragique dans quoi Andromaque n'entre pas puisque ses premiers mots sont : «Je passais jusqu'aux lieux où l'on garde mon fils ». Oui, glossaires et dictionnaires disent si peu de l'usage, et disent encore moins de l'usage ordinaire. Rien d'anormal, ce n'est pas leur fonction. Posséder un dictionnaire de puériculture est rarement d'une grande utilité devant un bébé qui pleure ou un ado qui ronchonne. Savoir comment fonctionne ce vocabulaire dans les usages ordinaires du monde, voilà ce qu'il serait utile et intéressant de décrire. Savoir comment fonctionnent ces vocabulaires des métaux rares, des finances, du «bio », des bâtis et bornes, de la médecine, de la génétique, etc. dans les usages ordinaires du monde, voilà ce qu'il serait utile et intéressant de décrire.

L'Encyclopédie de Diderot et d'Alembert offrira toujours un bon point de départ, même pour l'étude des vocabulaires de notre temps. En particulier, la présentation particulièrement rigoureuse et approfondie que donne l'édition numérique et critique ENCRRE, actuellement en cours. Mais au-delà, ou plus exactement au cœur de cet ouvrage, il y a ce que j'ai déjà signalé : les réflexions de Diderot sur le langage et la langue. Elles sont souvent, on le sait bien, en forme de questions ou de textes dialogués. Donc ouvertes au lecteur, comme les fins de Jacques le Fataliste. Et Jean-Pierre Seguin dans sa thèse sur le style de Diderot, si bien intitulée Diderot, le discours et les choses, a montré que ce style conduisait à « la présence de l'objet sensible » (1978:17). Il a montré que cela valait tout autant pour les mots concrets que pour les mots abstraits parce que le «style concret » de Diderot ramenait ces derniers eux aussi au «sensible » (1978: 36). Certes, il faut ici faire la part de la tonalité philosophique sensualiste, mais tout réduire au sensualisme, tel que le concevait Diderot ou tel que le concevait son siècle, serait revenir à un renfermement sur un univers mental. Il reste cependant, et c'est là une richesse de la réflexion et du style de Diderot, que ces objets « sensibles », visée du sensualisme, incluent d'avance les objets et les choses entendus comme nous les comprenons aujourd'hui.

C'est pourquoi, à l'approche de ma conclusion, la comparaison des noms chose et objet devient intéressante. Non pas parce qu'il arrive que chose et objet soient échangeables. Cela ne concerne que quelques cas : les choses perdues qui sont des « objets perdus » ou la part d'objets que comportent les choses corporelles, consomptibles, fongibles ou non de la langue du Palais. L'intérêt est dans le fait que chose a une définition que nous avons vue : « tout ce qui est et peut être appelé chose », alors qu'en regard objet n'a pas de définition et que c'est cette absence de définition qui lui permet d'être le nom objet. De fait, la diversité des emplois d'objet montre qu'il serait vain de chercher une valeur sémantique unique, même avec l'ajout d'une valeur figurée, tant il semble qu'il y ait plusieurs noms objet. L'objet des sens. L'objet de la seule vue, perçu par l'œil et étudié par l'optique. L'objet, cause d'une passion. L'objet, raison de la visée d'une action. L'objet, considéré par la critique dans son opposition à sujet pour dire «ce » qui est pensé comme distinct de l'acte de celui qui pense. L'objet, on pourrait dire « réifié » à la fin du XVIII ${ }^{\mathrm{e}}$ siècle quand la société industrielle et commerciale moderne impose un monde d'objets innombrables. Objet semble donc insaisissable. Pour autant, il est impossible de l'effacer dans la somme des qualifiants qui l'accompagnent parce qu'aucun d'eux ne capte objet à son profit. L'objet sonore, l'objet libidinal, l'objet du contrat, l'objet de la discussion, «l'unique objet de mon ressentiment » ou l'objet de ma passion, demeurent objet. Indéfinissable mais ineffaçable. Ce dernier caractère appartenant également au designatum d'Aristote ou à l'objet de Peirce.

Je cite une dernière fois Wittgenstein : «La signification d'un mot ne réside pas dans le fait que je peux me rendre présent son contenu (me le représenter intuitivement, en former une image hallucinatoire), mais dans le fait que je connais le chemin pour arriver à l'objet » (dans Bouveresse, $1976: 203$ ). Ce sont les interprétants qui balisent les chemins connus de chaque interlocuteur et si ces chemins convergent, c'est parce qu'ils ont le même horizon, l'horizon d'un même espace, un espace humain, un monde humain. À la différence des points de vue essentialistes et conventionnalistes qui s'accommodent sans problème de l'immanence de la langue, c'est l'usage appris et réglé dans une collectivité donnée qui nous permet de partager un monde qu'une logique d'immanence ne tient plus à l'écart.

Si la signification est le chemin pour arriver à l'objet, le mot et la chose sont liés par ce chemin. Considérer que la chose a fini par « exister», par exemple comme nous l'avons vu pour acier, marbre ou granit, n'oblige pas mener à fixer cette chose sous la forme d'un invariant. Elle n'est qu'un chemin parmi d'autres, celui de ce que nous appelons la science. Et cette science ne dispose d'aucun pouvoir pour donner sa bénédiction de baptême à l'invariant qu'elle semble susciter. Pas plus si la chose est là, très concrète. Le terme le plus « terme » devient un jour un simple mot. Souvenez-vous : «Tout ce qu'un symbole peut exprimer, il peut l'exprimer. » Sans cette absolue liberté d'expression, l'eau, un jour définie comme composée de deux atomes d'hydrogène et d'un atome d'oxygène, serait ainsi nommée. Nous dirions : Veux-tu de l'H2O dans ton vin ? De fortes précipitations d'H2O 
sont attendues ce soir sur l'Hérault. À moins de tomber sur un maniaque qui vous trouve ressembler à Georges Clooney ou Scarlett Johansson, selon les cas, comme deux gouttes d'oxyde d'hydrogène. Un conseil : Fuyez !

Si la chose n'existe pas, si elle est comme «en l'air » selon l'expression que j'ai employée pour les premiers emplois des mêmes acier, marbre et granit, si la chose n'existe pas, le chemin, lui, existe. Existe donc l'objet de la signification.

Je prends un exemple facile à saisir. Nous sommes aujourd'hui cernés par les normes et, dans nombre de leurs expressions, par le système décimal. Avant ces normes et ce système existaient point, pouce, ligne, pied, perche, lieue pour les longueurs, roquille, chopine, setier, feuillette, muid, pipe, conge ou queue pour les capacités. Et tant d'autres mesures! Toutes bien entendus différentes d'estimation d'une province à une autre, d'une ville à une autre, voire d'une rive à l'autre d'une rivière. Passer de ces mesures à nos mesures et à nos normes, cela n'a pas consisté simplement à passer d'un vocabulaire technique insuffisamment précis à un vocabulaire de termes précis, autrement dit : à passer d'un vocabulaire à une terminologie. Cela a signifié remplacer la négociation des mesures par l'acceptation de la normalisation. Cela a donc signifié : changer de monde. Non pas créer un monde, encore moins créer le monde, mais simplement en changer. Ceux qui ont applaudi ou qui applaudissent aujourd'hui, sont des gens « éclairés ». "Éclairés » comme je l'ai dit précédemment des «bien portants » invitant à remonter la pente. À l'encontre, comme l'écrit Roland Eluerd à propos des mesures de l'ancienne sidérurgie : «on peut imaginer sans peine qu'au pied de chaque fourneau, charbonniers, muletiers, chargeurs et fondeurs s'y retrouvaient très bien et que tous savaient parfaitement qui volait qui, avant d'être volé lui-même » (1993: 181).

Je ne voudrais pas qu'on pense que mes choix quasi systématiques de vocabulaires techniques ou similaires, signifient que ce j'ai cru pouvoir dire ne s'applique qu'à ces vocabulaires. Si les choses comme "en l'air » sont présentes dans ces vocabulaires, à plus forte raison quand il s'agit de mots comme bonheur, espoir, amour, liberté ou vertu que nous avons rencontré il y a peu. En étudiant le champ notionnel de l'ingénuité aux XVII ${ }^{\mathrm{e}}$ et XVIII siècles, Françoise Berlan étudiait à l'évidence le vocabulaire d'un univers mental, celui d'un sensible mondain au sens où mondain désigne des rapports de société. Mais elle a également rendu compte d'un sensible mondain au sens où mondain désigne des choses du monde, le contexte mondain. Et la présence du monde se rappelait à l'analyse puisque, écrit-elle, son analyse concerna finalement «beaucoup plus des valeurs de sens qu'une signification intangible, inhérente aux mots eux-mêmes » (1994:2). Soit l'exemple, là aussi, d'une lexicologie soucieuse certes de compréhension et d'explication, mais plus encore d'intercompréhension et de description.

J'arrive au terme de cette promenade lexicale et lexicographique sans itinéraire de théorie ou de rhétorique dont je mesure qu'elle a pu sembler plus une errance qu'un itinéraire. On me pardonnera cette facilité, mais au terme de cette errance, je m'arrête sur fauteuil.

Là encore, quel emblème de l'immanence de la langue ! Nous avons tous appris et enseigné que dans l'analyse logique du signifié du sémème fauteuil, telle que présentée par Bernard Pottier, se retrouvaient six sèmes : 1. pour s'asseoir, 2. sur pieds, 3. pour une personne, 4. avec dossier, 5. avec bras, 6. matériel rigide (1963: 13). L'intérêt de cette approche sémantique et de ses conséquences sémiologiques et lexicographiques ont été si importants! Mais ce que je veux retenir ici dans mon entêtement à garder le monde avec les mots, c'est d'abord noter que Bernard Pottier lui-même y était attentif. N'a-t-il pas en effet écrit : « Ce qui serait étonnant serait l'arbitraire du choix des sèmes par rapport au monde perceptible » (1974:59)?

Alors je m'engouffre dans l'étonnement de ces choix non arbitraires pour considérer quelques fauteuils si fatigués par les ans qu'ils n'ont plus de dossier, ou qu'ils n'ont plus de bras, ou qu'ils n'ont plus qu'un seul bras. Trop défaits pour des soldes intéressants, à peine bons pour un vide-grenier. Soit, mais, comme le demande MarieFrance Mortureux, dans son ouvrage au titre si juste La lexicologie entre langue et discours : " Comment se faitil que l'on continue, le cas échéant, à les appeler fauteuil ? » (1997 : 94)

À celle ou celui qui passera devant eux, imaginons qu'on propose de s'asseoir dans un ces fauteuils. Peu de chance qu'ils acceptent. «Pourquoi donc ? demandera-t-on. Sans dossier ou sans bras, ils ne méritent plus d'être appelés des fauteuils ? - La question n'est pas là, répondrait-on sans doute. » Il est vrai que ces chalands ne seraient sans doute pas des linguistes, qu'ils ne se poseraient pas ces étranges questions que les linguistes aiment examiner sans toujours s'apercevoir que le problème naît parfois de la question elle-même. Il est donc probable que leur réponse serait plutôt: « $\mathrm{Si}$, ce sont toujours des fauteuils, mais comment s'y asseoir? Ces fauteuils ne sont plus confortables. »

«Confort » : voilà une valeur sémantique majeure de fauteuil (Cottez, 1982 : 53). Une valeur qui vaut également pour gagner dans un fauteuil. Une valeur qui assure la présence du monde perceptible, une valeur que ne peuvent faire paraître des analyses ramenées à un schéma descriptif, cependant indispensable, ni réduites aux relations logiques d'implication et d'inclusion parce qu'elles passent "à côté du double fonctionnement référentiel et sémiotique de toute forme lexicale » (Tamba, $1998: 88)$. 
Et à qui douterait encore de la nécessaire présence du monde pour comprendre et partager le sens de fauteuil, on peut proposer de relire la belle phrase de Georges Perec, à la fin de son roman Les Choses, quand Jérôme et Sylvie deviennent presque riches : "Ils auront leur divan Chesterfield, leurs fauteuils de cuir naturel souples et racés comme des sièges d'automobile italienne... » La critique du moment a souvent présenté Les Choses comme une dénonciation de la société de consommation. Étrange lecture. Si dénonciation il y a, elle porte sur l'usage des mots quand on croit qu'ils ne sont que des mots qui ne sont pas des choses.

Perec n'a pas écrit un traité de sociologie politique, il a écrit un roman. Il n'a pas expliqué, il a narré et il a décrit. Ce ne sont pas tâches indignes de la lexicologie. Une lexicologie qui répondrait à la remarque implicitement injonctive de Diderot, une lexicologie qui, sans écarter la tâche « de prononcer qu'une chose est telle », accepterait « la nécessité de déterminer ce que c'est qu'être tel.»

\section{Bibliographie}

BASSEREAU J.-F., CHARVET-PELLO R. (2011). Dictionnaire des mots du sensoriel. Paris : Éditions Tec\&Doc. BASTUJI J. (1982). Structures des relations spatiales dans quelques langues naturelles. Introduction à une théorie sémantique. Paris-Genève : Droz.

BERLAN-LACOURT F. (1994). Le champ notionnel de l'ingénuité aux XVII et XVIII siècles. Université de Poitiers.

BERTIN T. (2018). La polysémie des noms de parties du corps humain en français. Analyse sémantique de artère, bouche, cœur, épaule et pied. Normandie Université.

BOUVERESSE J. (1976). Le Mythe de l'intériorité. Paris : Les Éditions de Minuit

COTTEZ H. (1982). Un va-et-vient entre l'homme et la nature. Propositions pour une lecture anthropologique du lexique français. Raison présente, 62, 39-55.

DELEDALLE G. (1990). Lire Peirce aujourd'hui. Bruxelles : Éditions Universitaires, De Boeck Université.

DELEDALlE G. (2000). Charles S. Peirce's Philosophy of signs. Bloomington : Indiana University Press.

DUBOIS J. (1962). Le vocabulaire politique et social en France de 1869 à 1872 à travers les ouvres des écrivains, les revues et les journaux. Paris : Larousse.

DUCROT O. (1972). Dire et ne pas dire. Principes de sémantique linguistique. Paris : Hermann.

ELUERD R. (1992). Note sur fibre et molécule dans Le Neveu de Rameau, L'information grammaticale, 52, 1416.

ELUERD R. (1993). Les Mots du fer et des Lumières. Contribution à l'étude du vocabulaire de la sidérurgie française (1722-1812). Paris : Honoré Champion, Genève : Slatkine.

ELUERD R. (2000). La Lexicologie, Paris : PUF.

GUILBERT L. (1965). La Formation du vocabulaire de l'aviation. Paris : Larousse.

GUILBERT L. (1967). La Formation du vocabulaire de l'astronautique. Université de Rouen : Larousse.

KLEIBER G. (1999). Problèmes de sémantique. La polysémie en question. P.U. du Septentrion : Villeneuve

d'Asq.

LERAT P. (1995). Les langues spécialisées. Paris : PUF.

MARTIN R. (1985). Aspect de la phrase analytique, Langages, 79, 40-54.

MATORÉ G. (1983), La Méthode en lexicologie, Paris, Didier, 2 éd.

MONK Ray (1993). Wittgenstein. Le devoir de génie, trad. Abel Gerschenfeld, Paris : Éditions Odile Jacob.

MORTUREUX M.-F. (1997). La lexicologie entre langue et discours. Paris : SEDES.

PEIRCE C. S. (1978). Écrits sur le signe, rassemblés, traduits et commentés par Gérard Deledalle. Paris : Éditions du Seuil.

POTTIER B. (1963). Recherches sur l'analyse sémantique en linguistique et en traduction mécanique, Nancy, Université.

POTTIER B. (1974), Linguistique générale. Théorie et description. Paris : Hachette.

SAUSSURE F. de (1972). Cours de linguistique générale, édition de Tullio De Mauro. Paris : Payot.

SEARLE J. R. (1982). Sens et Expression. Études de théories des actes de langage, trad. et préface de J. Proust. Paris : Éditions de Minuit.

SEGUIN J.-P. (1978) Diderot, le discours et les choses. Essai de description du style du philosophe en 1750. Paris : Klincksieck.

TAMBA-MECZ I., VEYNE P. (1979). Métaphore et comparaison selon Aristote, Revue des Études grecques, 92, Paris, 77-98.

TAMBA I. (1983). La composante référentielle dans un manteau de laine et un manteau en laine, Langue française, 57, 119-128.

TAMBA I. (1998). La Sémantique. Paris : PUF.

WAGNER R.-L. (1970). Les vocabulaires français II. Paris : Didier. 
WEXLER P. (1955). La Formation du vocabulaire des chemins de fer en France (1778-1862). Genève-Lille : Droz- Giard.

WITTGENSTEIN L. (1961). Tractatus logico-philosophicus suivi de Investigations philosophiques, trad. de l'allemand par Pierre Klossowski. Paris : Gallimard.

WITTGENSTEIN L. (1980). Grammaire philosophique, éd. posthume établie par Rush Rhees, trad. de l'allemand par Marie-Anne Lescouret. Paris : N.R.F.

${ }^{i}$ Les Faux-monnayeurs, Paris, Gallimard, $1925: 449$.

ii Sur les liens entre Peirce et Wittgenstein, voir Deledalle, $2000: 138,153$.

iii « Du dictionnaire à la forme schématique et retour? Le cas des verbes au radical en -lever. » Article encore sous l'anonymat de l'évaluation du comité de lecture de la revue au moment où j'écris ces lignes.

iv Jarrige F., Au temps des “tueuses de bras". Les bris de machines à l'aube de l'ère industrielle (1780-1860), Coll. Carnot, Presses Universitaire de Rennes, 2009.

${ }^{\vee}$ Alain, Propos de littérature, Paris, Éditions Gonthier, 1934 : 17-18.

vi Diderot, Le Neveu de Rameau, éd. J. Fabre, Genève, Droz, 1963 : 88-89 et 99.

vii Diderot, Euvres complètes, édition DPV, XVII, Paris, Hermann, $1987: 145$.

viii Le Journal d'un poète, in Euvres complètes, t. II, texte présenté et commenté par F. Baldensperger, Paris, Gallimard, Bibliothèque de la Pléiade, 1948 : 1180.

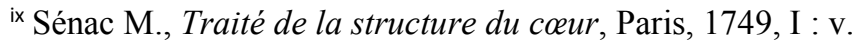

× Diderot, Euvres, tome IV, Esthétique - Théâtre, éd. Laurent Versini, Paris, Robert Laffont, coll. « Bouquins », $1996: 1124$.

xi Séance du 16 novembre 2019. «L'Intelligence Artificielle nous aide-t-elle à comprendre l'activité de langage ? » À paraître dans les Actes 2019 de la SLP.

xii Introduction à l'étude du vocabulaire médical (1600-1710), Besançon-Paris, Les Belles Lettres, 1955. 